\title{
Review of: "A meta-analysis of the effects of glucagon-like-peptide 1 receptor agonist (GLP1-RA) in nonalcoholic fatty liver disease (NAFLD) with type 2 diabetes (T2D)"
}

\author{
Ashwani Singal
}

Potential competing interests: The author(s) declared that no potential competing interests exist.

Dr Ghosal et al in this meta-analysis of eight randomized controlled trials show beneficial effects of GLP-1 analogues in patients with NAFLD and T2DM on body weight, metabolic parameters, liver fat, liver histology, and liver chemistry. Although, this is the first meta-analysis of its kind for which the authors need to be congratulated, there are some limitations of this meta-analysis which deserve a mention. Some of these are acknowledged by the authors especially on small number of studies evaluating liver histology. Further, authors may like to clarify the definition of biopsy resolution. This reviewer believes that authors are referring to resolution of Nash but would like to clarify this. Moreover, different interventions in active arm (different GLP-1 analogues), in control arm (placebo, SOC, another medication), and different imaging tools to measure liver fat in different studies limit the generalizability and strength of the study findings. Clearly, putting aside these limitations, this study is an important addition to the literature. Hopefully, the encouraging signals of GLP-1 analogues will be tapped by researchers and trialists in Nash with a larger multi center phase-3 study overcoming limitations identified here to establish the role of GLP-1 in routine clinical practice in the management of patients with T2DM and Nash. 